\title{
The Immunopathogenesis of Periodontal Disease
}

\section{- The Biological Basis for Treatment-}

\author{
Gregory J. Seymour BDS MDSc PhD MRCPath \\ Associate Professor of Periodontology \\ Director, Immunopathology Unit \\ Department of Social and Preventive Dentistry \\ The University of Queensland
}

The traditional approach to periodontal treatment planning has stemmed from the concept that the disease process is inevitably perpetuated by the presence of Periodontal pocketing, bony defects, furcation and mucogingival involvements, root surface changes and the occlusion. Current understanding of the nature of chronic inflammatory periodontal disease challenges this traditional approach to therapeutic assessment.

In recent years it has become evident that at least two forms of chronic inflammatory periodontal disease exist. One of these, termed the 'stable' lesion, is relatively inactive and does not lead to tooth loss. The other, termed the 'progressive' lesion, leads ultimately to tooth loss and endangers the life of the dentition. It has also become apparent that chronic inflammatory periodontal disease follows a cyclical pattern, with periods of destruction interspersed with periods of stability. A number of concepts involving 'bursts' of activity have been advocated but the nature and length of these bursts remains controversial. Immunohistological studies have shown that the stable lesion is dominated by T-cells while the progressive lesion is dominated by B-cells and plasma cells. This observation has lead to the development of a model of disease based on the conversion of the $\mathrm{T}$-cell response to one involving B-cells.

This seminar will also show how this model represents the biological basis for treatment. It will also show how the model can be used to study immunoregulatory control mechanisms. Experimental gingivitis studies enabled the identification of T-cell subsets and their expression of activation markers to be made. These revealed that the $\mathrm{CD} 4: \mathrm{CD} 8$ ratio in the stable lesion is $2: 1$ while extraction of cells from periodontitis lesions revealed a $1: 1$ ratio. This data suggested a $\mathrm{T}$-cell/M $\phi$ immunoregulatory imbalance may exist in the progressive lesion.

Further functional data supported this concept, while CD 4 subset identification showed a dominance of activated memory $\mathrm{T}$-cells in the lesion. Limit dilution analysis of peripheral blood T-cells showed the presence of periodontopathic bacteria specific T-cells supporting the concept of antigen specific T-cell control in periodontal disease. This data will be reviewed within the context of the disease model and the concept of non specific versus antigen specific B-cell activation will be explored. 